\title{
Augmented Reality Assisted Laparoscopic Partial Nephrectomy
}

\author{
Adrian Schneider, Simon Pezold, Andreas Sauer, Jan Ebbing, \\ Stephen Wyler, Rachel Rosenthal, and Philippe C. Cattin \\ Medical Image Analysis Center, University of Basel, Switzerland
}

\begin{abstract}
Computer assisted navigation is a widely adopted technique in neurosurgery and orthopedics. However, it is rarely used for surgeries on abdominal organs. In this paper, we propose a novel, noninvasive method based on electromagnetic tracking to determine the pose of the kidney. As a clinical use case, we show a complete surgical navigation system for augmented reality assisted laparoscopic partial nephrectomy. Experiments were performed ex vivo on pig kidneys and the evaluation showed an excellent augmented reality alignment error of $2.1 \mathrm{~mm} \pm 1.2 \mathrm{~mm}$.
\end{abstract}

Keywords: Augmented Reality, Electromagnetic Tracking, Navigation.

\section{Introduction}

Laparoscopic partial nephrectomy (LPN) is considered to be the standard of care for small renal tumors. As opposed to radical nephrectomy where the whole organ is removed, only parts of the kidney get extracted in partial nephrectomy. Although this nephron-sparing surgery is increasingly applied, it is still underused as was found by an investigation among 66000 patients undergoing radical or partial nephrectomy in the US [2]. In tumors smaller than $4 \mathrm{~cm}$ in diameter, LPN has shown to provide equivalent cancer control as compared to radical nephrectomy, but with the advantage of nephron-sparing [8]. The latter results in a higher renal performance and thus in a better quality of life for the patient.

One major challenge in LPN is obtaining optimal surgical margins; that is, removing all cancerous organ parts while keeping as much healthy tissue as possible. Established strategies to decrease the percentage of resections resulting in positive margins make use of intraoperative ultrasound and fresh frozen section analysis 8 . More recently, surgical support by accurate 3D navigation systems started to gain importance.

In this paper, we present a novel, non-invasive method for navigated kidney surgery. In particular, an electromagnetic tracking system is used to determine the kidney pose by localizing a tiny magnetic sensor within a catheter tip that was placed through the urinary passages (urethra, bladder, ureter) at the renal pelvis (Fig. 1, 2). To our knowledge, this transurethral renal access is used for the first time for tracking purposes. It became possible by applying miniaturized electromagnetic sensors that just recently appeared on the market.

P. Golland et al. (Eds.): MICCAI 2014, Part II, LNCS 8674, pp. 357-364, 2014.

(C) Springer International Publishing Switzerland 2014 


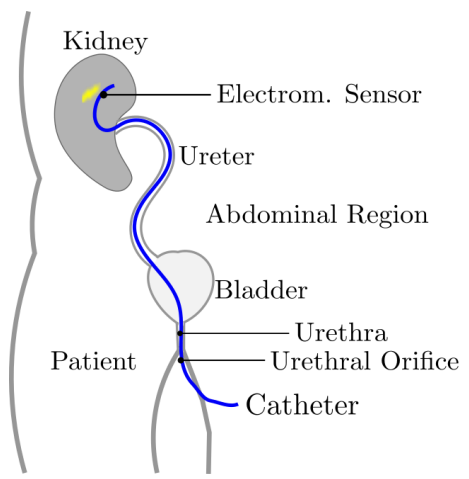

Fig. 1. Transurethral placement of the electromagnetic sensor in the renal pelvis of the right kidney

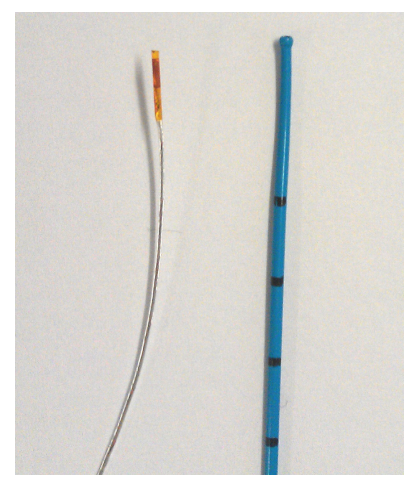

Fig. 2. Electromagnetic sensor, Model 90, Ascension Corp. (left). Ureteral catheter, Angiomed GmbH (right).

By additionally tracking the laparoscope, we implement a reliable surgical navigation system for LPN. To increase usability and to allow for a seamless integration into the surgical workflow, the classical abstract navigation view is extended with an intuitive augmented reality (AR) visualization technique.

In 2008, Nakamoto et al. 5] proposed a similar LPN guidance method, which uses the Calypso $4 D$ to determine the position of implanted wireless magnetic transponders. Additionally, an optical system was used to track the laparoscope. By referencing those two coordinate systems (CS), AR can be performed. An advantage of this approach is the robust tracking of the kidney, as the transponders are implanted and unlikely to shift. The downsides are its extraordinary high price of $>\$ 400000$, its large footprint, and the necessity of a second tracker. In our proposed approach, one electromagnetic tracker with wired sensors is sufficient, since the kidney transponder coils and the connecting wire can be packed into a single catheter. This provides us with the opportunity to apply electromagnetic tracking systems that are transportable, much cheaper $(<\$ 15000)$, and relatively robust to ferromagnetic disturbances. A further advantage of our method is that no transponders have to be implanted into the kidney.

Hughes-Hallett et al. 3 published an excellent review of different AR techniques to perform LPN. Besides the above-mentioned approach, it also describes fundamentally different methods.

\section{Materials and Methods}

The setup of the proposed navigation system is shown in Fig. 3. In the following, we are going to describe each part of the tracking pipeline in detail, covering deployed materials and algorithms. 


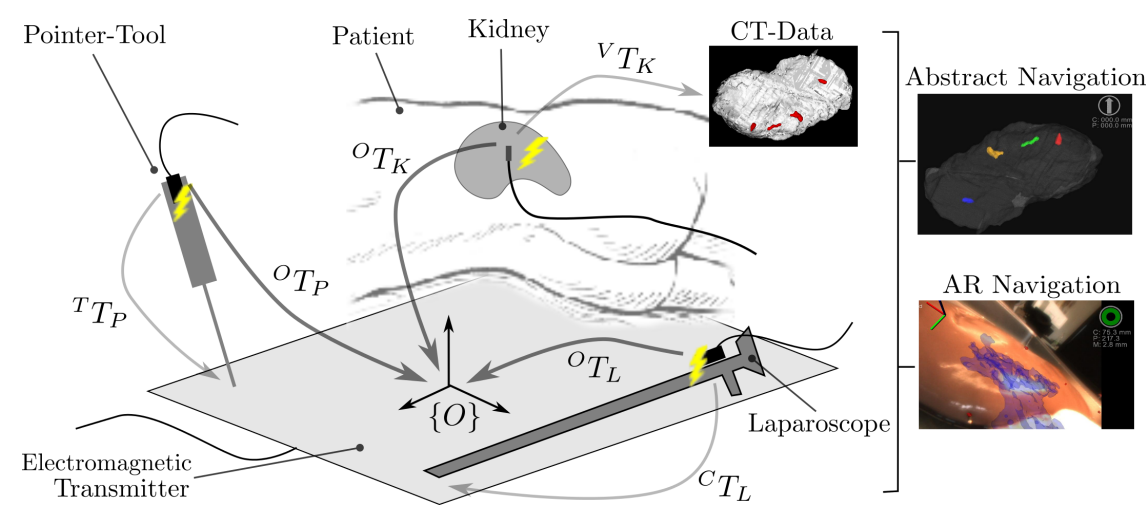

Fig. 3. Navigation system overview. Arrows denote affine transformations.

\subsection{Electromagnetic Tracker}

As an electromagnetic tracking device, the trakSTAR 2 together with the flat transmitter from Ascension Technology Corp., Shelburne, USA is used. The electromagnetic sensors support 6 degrees of freedom. Therefore, the rotation and translation of each sensor can be determined explicitly and are compactly represented by an affine homogeneous $4 \times 4$ transformation matrix. A Model 90 sensor with a diameter of $0.9 \mathrm{~mm}$ is built into the catheter. Model 800 sensors with a diameter of $7.9 \mathrm{~mm}$ are attached to the surgical tools. In an OR environment and in the presence of surgical tools (causing ferromagnetic disturbances), a dynamic accuracy of $1.3 \mathrm{~mm}$ and a static accuracy of $2.4 \mathrm{~mm}$ were determined for a similar setup [10].

As shown in Fig. 3, the electromagnetic tracking system provides the affine transformations ${ }^{O} T_{K}$ (kidney sensor $\rightarrow$ origin), ${ }^{O} T_{L}$ (laparoscope sensor $\rightarrow$ origin) and ${ }^{O} T_{P}$ (pointer-tool sensor $\rightarrow$ origin).

\subsection{Laparoscope}

We use the $30^{\circ}$ Hopkins2 laparoscope (Storz GmbH, Tuttlingen, Germany) together with the Prosilica GC laparoscopic camera (Allied Vision Technologies GmbH, Stadtroda, Germany). The intrinsic parameters of the laparoscopic optic are determined based on 2D-3D point-correspondences [9] established from a $5 \times 8$ chessboard pattern.

As shown in Fig. 3, the transformation ${ }^{C} T_{L}$ links the laparoscopic sensor's $\mathrm{CS}$ and the CS of the camera. The methods to determine ${ }^{C} T_{L}$ are known as hand-eye calibration. We implemented the least-squares approach proposed by Park et al. [7].

\section{$2.3 \quad$ Pointer-Tool}

A laparoscopic gripper manufactured by Covidien, Mansfield, USA is used as a navigated surgical device. The name pointer-tool is derived from its application 
for determining the $3 \mathrm{D}$ position of the tool-tip. The transformation ${ }^{T} T_{P}$ (Fig. 3) is the translation from the pointer-tool sensor to the pointer-tool tip. We compute it by the method described in 6 .

\subsection{Kidney Registration}

The kidney registration results in the transformation ${ }^{V} T_{K}$, which maps the CS of the virtual 3D data to the CS of the electromagnetic sensor in the kidney (Fig. 3). In the case of LPN, the 3D data are acquired from a pre-operative diagnostic CT scan.

A well established method to compute the registration is based on $3 \mathrm{D}-3 \mathrm{D}$ point correspondences [1. If applied to the situation in Fig. 3, one has to select $N \geq 3$ identifiable landmarks ${ }^{V} L_{n}(n=1, \ldots, N)$ from the virtual 3D data. By using the pointer-tool, the corresponding landmarks ${ }^{T} P_{n}$ are then probed on the real kidney. In order to represent them in the kidney sensor's CS, denoted as ${ }^{K} P_{n}$, the following linear transformation is applied:

$$
{ }^{K} P_{n}=\left({ }^{O} T_{K}\right)^{-1} \cdot{ }^{O} T_{P} \cdot\left({ }^{T} T_{P}\right)^{-1} \cdot{ }^{T} P_{n} \quad(n=1, \ldots, N) .
$$

In a next step, we compute the transformation ${ }^{V} T_{K}$ by using the two point sets ${ }^{V} L_{n}$ and ${ }^{K} P_{n}(n=1, \ldots, N)$ as input for the method in [1]. The residual fitting mismatch between the two $3 \mathrm{D}$ point sets is the average registration error

$$
E_{\mathrm{reg}}=\frac{1}{N} \sum_{n=1}^{N}\left(\left\|{ }^{V} L_{n}-\left({ }^{V} T_{K} \cdot{ }^{K} P_{n}\right)\right\|\right) .
$$

\subsection{Navigation}

After the kidney registration and device calibration, the remaining task for completing the surgical 3D navigation toolchain is to generate the navigation views.

The Classical Abstract Navigation View can be realized by transforming virtual anatomical $3 \mathrm{D}$ data ${ }^{V} D$ and the surgical tool ${ }^{T} P$ into a common CS and by displaying them in a suitable $3 \mathrm{D}$ environment. In our implementation, we chose the CS of the virtual $3 \mathrm{D}$ data as reference $\mathrm{CS}$. Therefore, ${ }^{V} D$ is already in the correct CS. The surgical tool needs to be transformed from ${ }^{T} P$ to ${ }^{V} P$ by

$$
{ }^{V} P={ }^{V} T_{K} \cdot\left({ }^{O} T_{K}\right)^{-1} \cdot{ }^{O} T_{P} \cdot\left({ }^{T} T_{P}\right)^{-1} \cdot{ }^{T} P .
$$

The Augmented Reality Navigation builds upon the classical abstract view. In addition, the position and rotation of the laparoscopic camera, ${ }^{C} T_{L}$, is mapped to the $3 \mathrm{D}$ environment's virtual camera pose ${ }^{V} C$ by

$$
{ }^{V} C={ }^{V} T_{K} \cdot\left({ }^{O} T_{K}\right)^{-1} \cdot{ }^{O} T_{L} \cdot\left({ }^{C} T_{L}\right)^{-1} .
$$

Furthermore, the projective properties of the virtual camera are aligned with those determined during the laparoscopic intrinsic camera calibration. Finally, the undistorted laparoscopic image is put as background into the virtual scene. 


\section{$3 \quad$ Experiments and Results}

For the following experiments, we used six pig kidneys. Four of them were prepared for the Sensor Shift experiment (Sec. 3.1) and two were dedicated to determine the Overall Navigation Error (Sec. 3.2). For comparison purposes, a rigid kidney-like mock object was taken into the experiments as well. The Mock is constructed using a sponge and holds an artificial tumor made of silicone.

\subsection{Sensor Shift}

In the presented tracking approach, it is key that the electromagnetic sensor placed in the renal pelvis does not move relative to the kidney while the organ is exposed to external mechanical forces and motion during mobilization. In addition, the error of applying a rigid registration to a soft-tissue structure needs to be evaluated.

In order to observe sensor shifts, we compared an initial 3D-3D registration ${ }^{V} T_{K_{0}}$ with subsequent registrations ${ }^{V} T_{K_{i}}$. Between each pair of consecutive registrations, we applied a standardized motion to the kidney, similar to the ones that can be observed during mobilization. The registration differences of the respective $4 \times 4$ homogeneous transformation matrices can then be split into a rotational part $\Delta \Theta_{i}$ and a translational part $\Delta t_{i}$. Since rotations are executed first, $\Delta t_{i}$ depends heavily on $\Delta \Theta_{i}$. At the same time, the distance to the CS origin matters. Therefore, we decided to consider only $\Delta \Theta_{i}$ as a quantitative measure between the registrations. A good illustration of the effect of $\Delta \Theta_{i}$ is the resulting point shift $\Delta P_{i}$ in a certain distance $d$ from the sensor. Since the length of an average kidney is about $10 \mathrm{~cm}$, it is reasonable to assume that the sensor can be placed within a range of $d<2.5 \mathrm{~cm}$ to the region of treatment.

We compute $\Delta \Theta_{i}$ by the inner product of unit quaternions 4 as

$$
\Delta \Theta_{i}=\arccos \left(\left|q\left(r\left({ }^{V} T_{K_{0}}\right)\right) \cdot q\left(r\left({ }^{V} T_{K_{i}}\right)\right)\right|\right),
$$

since this rotation metric uses the common unit of radians. Here, $q($.$) converts$ a rotation matrix into a $4 \times 1$ quaternion and $r($.) extracts the $3 \times 3$ rotation matrix from a registration. The shift of an arbitrarily chosen point, expressed as Euclidean distance, is calculated as

$$
\Delta P_{i}=\left\|r\left({ }^{V} T_{K_{0}}\right) \cdot \vec{D}-r\left({ }^{V} T_{K_{i}}\right) \cdot \vec{D}\right\| \quad \text { with } \quad \vec{D}=\frac{1}{\sqrt{3}} \cdot\left[\begin{array}{l}
1 \\
1 \\
1
\end{array}\right] \cdot d .
$$

The influence of the nonrigid part of the kidney is difficult to isolate. As a reference, the results of the rigid Mock can be used. An additional indicator is the registration error $E_{\text {reg. }}$. In principle, differences between the pre-operative CT scan and the actual kidney shape lead to an increased registration error.

The following experiment was performed ex vivo with four pig kidneys and the above-described Mock. In order to avoid registration errors introduced through landmark correspondence mismatches, five artificial landmarks with precisely 
known 3D coordinates from the $\mathrm{CT}$ data were used. We chose $20^{\circ}$ of rotation and $30 \mathrm{~mm}$ of translation relative to the renal hilum (i.e., the entrance of ureter and blood vessels to the kidney) as a reasonable parameterization for simulating the possible mobilization of the kidney during LPN. For every step $i$, the same motion sequence with the given values was applied to the organ. The repositioning error of the used pointer tool was $0.2 \mathrm{~mm}$.

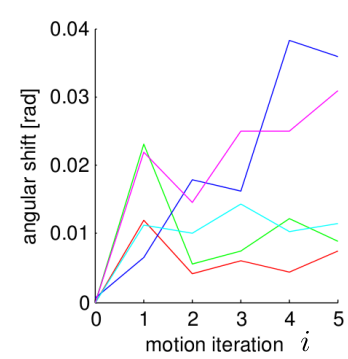

Fig. 4. Registration rotational shift $\Delta \Theta$

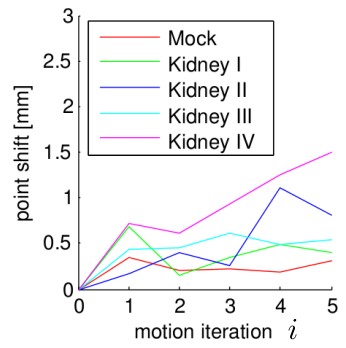

Fig. 5. Point shift $\Delta P$ for $d=25 \mathrm{~mm}$

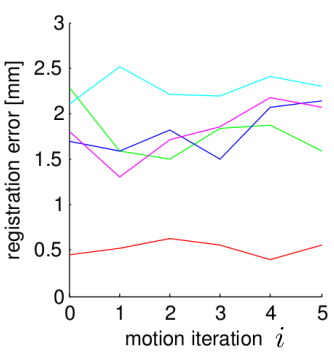

Fig. 6. Registration error $E_{\text {reg }}$

Results: As expected, the Mock performed best. Its registration errors (Fig. 6) are about four times smaller than those of the kidneys. The difference can clearly be attributed to the influence of organ deformation or, in general terms, to nonrigidity.

In terms of the sensor shift, the Mock and kidneys perform comparably. The rotational shifts (Fig. (4) of Kidney $I I$ and $I V$ stand out, and so do the corresponding point shifts (Fig. 5). In the worst case, an error of $1.5 \mathrm{~mm}$ can be expected in a distance of $25 \mathrm{~mm}$ from the sensor after applying the motion sequence five times.

In this experiment, the registration error is considerably larger than the point shift. This is the case because the registration is performed over the whole kidney $(d \approx 50-100 \mathrm{~mm})$, whereas the point shift is estimated for a distance of $d=$ $25 \mathrm{~mm}$ from the electromagnetic sensor.

\subsection{Navigation Error}

In the following experiment, we determined the overall positioning accuracy of our navigation system in both modes: abstract navigation view and AR. The experiment was performed on two ex-vivo pig kidneys and the Mock. For each subject, 20 measurements were taken at five known artificial landmarks distributed over the whole kidney. However, in order to be close to the clinical application, the kidney registration was performed using four well identifiable natural landmarks. The repositioning error of the used pointer tool was $0.2 \mathrm{~mm}$, 


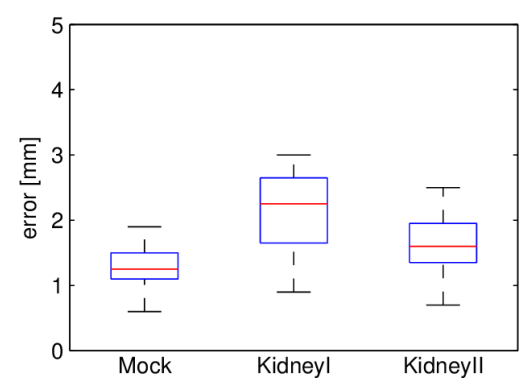

Fig. 7. Navigation error $\Delta_{\text {Abstract }}$

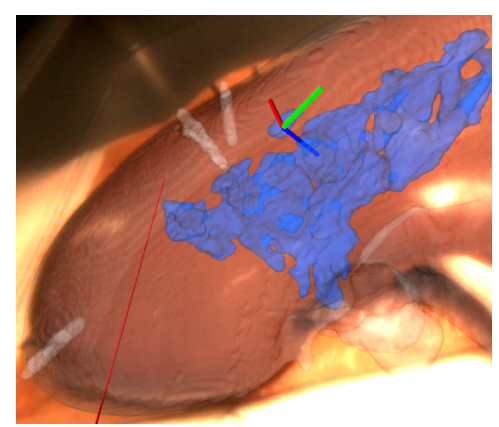

Fig. 9. Real kidney overlaid with virtual renal pelvis (blue)

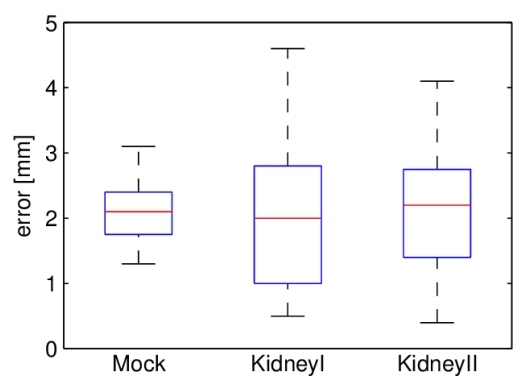

Fig. 8. Navigation error $\Delta_{\mathrm{AR}}$

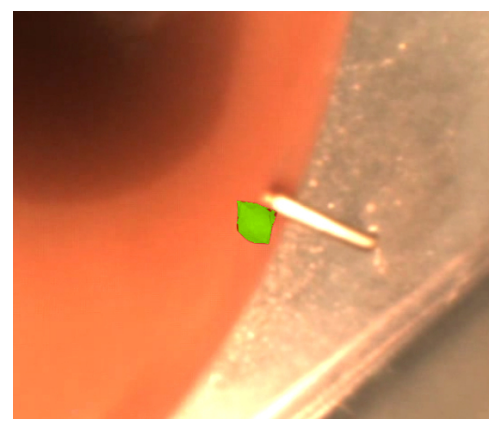

Fig. 10. Virtual marker (green) beside its real corresponding landmark

the error of the intrinsic camera calibration was 0.5 pixel, and the error of the hand-eye calibration of the laparoscopic camera was measured to be $0.4 \mathrm{~mm}$.

In the case of the abstract navigation view, the pointer tool was used to probe a defined landmark $a$ on the kidney and to compare its position ${ }^{T} P_{a}$ against the known 3D location ${ }^{V} L_{a}$. The difference is the target registration error $\Delta_{\text {Abstract }_{a}}$ :

$$
\Delta_{\text {Abstract }_{a}}=\left\|{ }^{V} L_{a}-\left({ }^{V} T_{K} \cdot\left({ }^{O} T_{K}\right)^{-1} \cdot{ }^{O} T_{P} \cdot\left({ }^{T} T_{P}\right)^{-1} \cdot{ }^{T} P_{a}\right)\right\| .
$$

The target registration error of the $\mathrm{AR}$ navigation, $\Delta_{\mathrm{AR}_{a}}$, was determined by comparing the visualized location of a landmark on the laparoscopic image stream against its true position (Fig. 10):

$$
\Delta_{\mathrm{AR}_{a}}=\left\|^{T} P_{R_{a}}-{ }^{T} P_{V_{a}}\right\|
$$

In practice, we used the pointer-tool tip to probe the $3 \mathrm{D}$ position of a visualized landmark projected onto the kidney surface ${ }^{T} P_{V_{a}}$ and to probe the true position ${ }^{T} P_{R_{a}}$. The distance between the laparoscope and a particular landmark was between $20 \mathrm{~mm}$ and $35 \mathrm{~mm}$.

Results: Registration errors are $1.0 \mathrm{~mm}$ for the Mock, $2.3 \mathrm{~mm}$ for Kidney I, and $1.8 \mathrm{~mm}$ for Kidney II. For the kidneys, the determined mean error is $1.9 \mathrm{~mm}$ 
$(s t d=0.6 \mathrm{~mm})$ for abstract navigation (Fig. 7) and $2.1 \mathrm{~mm}(s t d=1.2 \mathrm{~mm})$ in AR mode (Fig. 8, 9, 10).

The error of the AR navigation is higher than the one of the abstract navigation, which can be explained by the additional error of the camera transformation. The results also show that the standard deviation of the AR system is much higher. This might be caused by the intrinsic camera parameters. We observed wide-spreading errors of one landmark while changing viewing positions.

\section{Conclusion}

We showed that our transurethral electromagnetic tracking approach can be applied for LPN, which uses resection margins of 5-7 mm. With an AR error range of $0.9-3.3 \mathrm{~mm}$, our approach performs better than the Calypso based LPN [5] $(3-5 \mathrm{~mm})$. However, the experiments also showed that our method is prone to sensor shifts under possible mobilization of the kidney during surgery. For the moment, this issue is tackled by performing re-registrations. In the future, we hope to avoid it by using a dedicated catheter shape.

\section{References}

1. Arun, K.S., Huang, T.S., Blostein, S.D.: Least-squares fitting of two 3-d point sets. IEEE Trans. on Pattern Analysis and Machine Intelligence (5), 698-700 (1987)

2. Hollenbeck, B.K., Taub, D.A., Miller, D.C., et al.: National utilization trends of partial nephrectomy for renal cell carcinoma: a case of underutilization? Urology 254-259 (2006)

3. Hughes-Hallett, A., Mayer, E.K., Marcus, H.J., Cundy, T.P., Pratt, P.J., Darzi, A.W., Vale, J.A.: Augmented reality partial nephrectomy: Examining the current status and future perspectives. Urology (2013)

4. Huynh, D.Q.: Metrics for 3d rotations: Comparison and analysis. Journal of Mathematical Imaging and Vision 35(2), 155-164 (2009)

5. Nakamoto, M., Ukimura, O., Gill, I., Mahadevan, A., Miki, T., Hashizume, M., Sato, Y.: Realtime organ tracking for endoscopic augmented reality visualization using miniature wireless magnetic tracker. In: Dohi, T., Sakuma, I., Liao, H. (eds.) MIAR 2008. LNCS, vol. 5128, pp. 359-366. Springer, Heidelberg (2008)

6. Onprasert, O.,, S.: A novel method on tool tip calibration for biomedical application. In: The World Congress on Computer Science and Information Engineering, pp. 650-653 (2011)

7. Park, F.C., Martin, B.J.: Robot sensor calibration: solving $a x=x b$ on the euclidean group. IEEE Trans. on Robotics and Automation 10(5), 717-721 (1994)

8. Weise, E.S., Winfield, H.N.: Laparoscopic partial nephrectomy. J. Endourol. 19, 634-642 (2005)

9. Zhang, Z.: A flexible new technique for camera calibration. IEEE Transactions on Pattern Analysis and Machine Intelligence 22(11), 1330-1334 (2000)

10. Zhou, J., Sebastian, E., Mangona, V., Yan, D.: Real-time catheter tracking for highdose-rate prostate brachytherapy using an electromagnetic 3d-guidance device: A preliminary performance study. Medical Physics 40(2), 021716 (2013) 